REVISTA MATEMÁTICA de la Universidad Complutense de Madrid Volumen 9, número suplementario, 1996 http://dx.doi.org/10.5209/rev_REMA.1996.v9.17531

\title{
A unified approach for commutators theorems in interpolation theory, a survey ${ }^{1}$.
}

\author{
M. J. CARRO, J. CERDÀ and J. SORIA
}

\begin{abstract}
We review the main facts that are behind a unified construction for the commutator theorem of the main interpolation methods.
\end{abstract}

\section{Introduction}

Complex and real interpolation methods are the abstract counterpart of Riesz-Thorin and Marcinkiewicz theorems. They associate to every couple $\bar{A}=\left(A_{0}, A_{1}\right)$ of compatible Banach spaces a scale of new norms or spaces:

Recall that, for the Calderón's complex method, a Banach space $\mathcal{F}(\bar{A})$ is considered. It is the space of all bounded continuous functions on the strip $\overline{\mathbf{S}}=\{0 \leq \Re z \leq 1\}$,

$$
F: \overline{\mathbf{S}} \longrightarrow A_{0}+A_{1}=\Sigma(\bar{A}) \text {, }
$$

which are analytic on $\mathrm{S}=\{0<\Re z<1\}$ and such that $F_{j}(t)=$ $F(j+i t)$ define two continuous functions $F_{j}: \mathbf{R} \longrightarrow A_{j}$ with the property $\lim _{t \rightarrow \infty}\left\|F_{j}(t)\right\|_{j}=0$, where we denote $\|\cdot\|_{j}=\|\cdot\|_{A_{j}}(j=0,1)$. The norm on $\mathcal{F}(\bar{A})$ is

$$
\|F\|_{\mathcal{F}}=\max _{j=0,1}\left(\sup _{t \in \mathbf{R}}\left\|F_{j}(t)\right\|_{j}\right)
$$

AMS Subject Classification: $46 \mathrm{M} 35,46 \mathrm{E} 30$.

Servicio Publicaciones Univ. Complutense- Madrid, 1996.

${ }^{1}$ This work has been partially supported by DGICYT, Grant PB940897. 
and, for every $0<\theta<1$, we have the interpolated space

$$
[\bar{A}]_{\theta}=\{F(\theta) ; F \in \mathcal{F}(\bar{A})\}
$$

with the norm $\|x\|_{[\theta]}=\inf \left\{\|F\|_{\mathcal{F} ; F(\theta)=x\}}\right.$.

In [RW], Rochberg and Weiss considered the rate of change of these interpolated norms by analyzing estimates of the derivatives $F_{x}^{\prime} 0(\theta)$ when $F_{x}$ is almost optimal for $x \in[\vec{A}]_{\theta}$, in the sense that $F_{x} \in \mathcal{F}(\bar{A})$, $F_{x}(\theta)=x$ and $\left\|F_{x}\right\|_{\mathcal{F}} \simeq\|x\|_{[\theta]}$. A corresponding study for the real method was carried out by Jawerth, Rochberg and Weiss in [JRW].

In both cases, interpolation theorems are derived for commutators of bounded linear operators with certain operators $\Omega$, generally nonlinear and unbounded, with nice applications to classical analysis. For a useful survey we refer to [CJMR].

In [CCS1], the authors have shown that both are in fact special cases of a general construction where the main ideas of the proofs appear very neately. Moreover, this construction gives rise to other commutator theorems, such that those included in [CCMS] for Lions-Schechter methods.

Here we review (with almost no proofs but with complete references) the main facts concerning this unified approach. As in [RW], we start by considering what it happens when in Riesz-Thorin theorem we compare the derivatives of the functions that appear along the proof with those of certain modifications of these functions. This is of use to state how cancelation, optimal selection and a second interpolation method are involved.

For the sake of simplicity, we consider the "diagonal case". It states that if

$$
T:\left(L^{p_{0}}(\lambda), L^{p_{1}}(\lambda)\right) \longrightarrow\left(L^{p_{0}}(\mu), L^{p_{1}}(\mu)\right)
$$

is linear and bounded $\left(\|T f\|_{p_{j}} \leq M_{i}\|f\|_{p_{j}}\right)$ and $p_{0}<p<p_{1}$, then $T: L^{p}(\lambda) \longrightarrow L^{p}(\mu)$ (with constant $M \leq M_{0}^{1-\theta} M^{\theta}$ if $1 / p=(1-\theta) / p_{0}+$ $\left.\theta / p_{1}\right)$, i.e., for any simple function $f$ such that $\|f\|_{p}=1$,

$$
\left|\int g(T f) d \mu\right| \leq M . \quad\left(g \text { simple and }\|g\|_{p^{\prime}}=1\right) .
$$

In Thorin's proof this estimate is obtained as an application of the threelines theorem to the function

$$
F(z)=\int g_{z}\left(T f_{z}\right) d \mu
$$


with

$$
f_{z}=|f|^{\alpha(z)} \operatorname{sgn} f, \quad g_{z}=|g|^{(1-\alpha(z)) p^{\prime}} \operatorname{sgn} g, \quad \alpha(z)=\frac{1-z}{p_{0}}+\frac{z}{p_{1}}
$$

(hence $p=1 / \alpha(\theta)$ and $F(\theta)=\int g(T f) d \mu$ ). Let also

$$
G(z)=\int g_{z}(T f)_{z} d \mu
$$

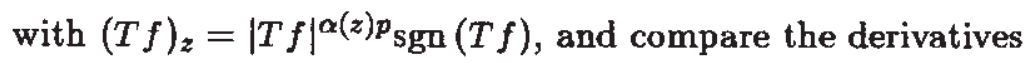

$$
\begin{gathered}
F^{\prime}(\theta)=\int\left[\left(\frac{p^{\prime}}{p_{0}}-\frac{p^{\prime}}{p_{1}}\right)(g \log |g|) T f-\left(\frac{p}{p_{0}}-\frac{p}{p_{1}}\right) g T(f \lg |f|)\right] d \mu \\
G^{\prime}(\theta)=\int\left[\left(\frac{p^{\prime}}{p_{0}}-\frac{p^{\prime}}{p_{1}}\right)(g \log |g|) T f-\left(\frac{p}{p_{0}}-\frac{p}{p_{1}}\right) g T(f) \lg |T f|\right] d \mu .
\end{gathered}
$$

If we denote $L h=h \log |h|$, we obtain

$$
G^{\prime}(\theta)-F^{\prime}(\theta)=\left(\frac{p}{p_{0}}-\frac{p}{p_{1}}\right) \int g\{T(L f)-L(T f)\} d \mu
$$

where, for the circle $\gamma=\{|z|=r\}$ with $r=d(\theta, \partial \mathrm{S})$,

$$
\left|F^{\prime}(\theta)\right|=\left|\frac{1}{2 \pi i} \int_{\gamma} \frac{F(z)}{(z-\theta)^{2}} d z\right| \leq \frac{M}{r}, \quad\left|G^{\prime}(\theta)\right| \leq \frac{M}{r} .
$$

From (2) and (3) it follows that

$$
\|[T, L] f\|_{p} \leq C \quad\left(\|f\|_{p}=1\right) .
$$

Although $L$ is not homogeneous, for the commutator $[T, L]=T L-L T$ we have $[T, L](\lambda f)=\lambda[T, L] f$ and (4) is equivalent to

$$
\|[T, L] f\|_{p} \leq C\|f\|_{p} .
$$

For the homogeneous operator

$$
\Omega h=h \log \frac{|h|}{\|h\|_{p}},
$$

from Riesz-Thorin theorem and from (5) we obtain

$$
\left\|[T, \Omega]_{f}\right\|_{p} \leq C\|f\|_{p},
$$

which is the commutator theorem.

Around (7) several remarks are to be pointed out: 
1. With evaluation $\delta_{\theta}: F \mapsto F(\theta)$, evaluation of the derivatives, $\delta_{\theta}^{\prime}: F \mapsto F^{\prime}(\theta)$, is used.

2. $\delta_{\theta}$ and $\delta_{\theta}^{\prime}$ are combined through cancelation property (2).

3. $\delta_{\theta}$ and $\delta_{\theta}^{\prime}$ can be used in the abstract frame of Calderón's method (1) if we take an almost optimal function $F_{x}$ for every $x \in[\bar{A}]_{\theta}$.

4. For concrete examples, an almost optimal election is done to identify the interpolated spaces, such as $\left[L^{p_{0}}, L^{p_{1}}\right]_{\theta}=L^{p}$. In this example $\Omega x=\delta_{\theta}^{\prime}\left(F_{x}\right)$.

For undefined notation and standard definitions of interpolation theory we refer to [BL]. We write $A \simeq B$ if $A \leq B$ and $B \leq A$ up to a multiplicative constant.

\section{Interpolators and commutator theorem}

With Calderón's method as a model, we define an interpolator $\Phi$ over spaces $H(\bar{A})$ (the function spaces) as a couple $(H, \Phi)$ where $H$ is a functor from a class $\mathcal{C}$ of compatible couples of Banach spaces to normed spaces,

$$
H: \bar{A} \mapsto H(\bar{A}), \quad H: \mathcal{L}(\bar{A} ; \bar{B}) \mapsto \mathcal{L}(H(\bar{A}) ; H(\bar{B})),
$$

and $\Phi$ is a family of bounded linear operators

$$
\Phi_{\bar{A}} \in \mathcal{L}(H(\bar{A}) ; \Sigma(\bar{A})) \quad(\bar{A} \in \mathcal{C})
$$

such that

$$
T \Phi_{\bar{A}}=\Phi_{\bar{B}} H(T) \quad(T \in \mathcal{L}(\bar{A} ; \bar{B})) .
$$

Remark By $T \in \mathcal{L}(\bar{A} ; \bar{B})$ we mean that $T$ is an interpolation operator, i.e.,

$$
T \in \mathcal{L}(\Sigma(\bar{A}) ; \Sigma(\bar{B})), T\left(A_{j}\right) \subset A_{J},\|T\|=\max _{j=0,1}\|T\|_{A_{j}, B_{j}}<\infty .
$$

Since $\|a\|_{\Sigma(\bar{A})}=\inf _{a=a_{0}+a_{1}}\left\|a_{0}\right\|_{0}+\left\|a_{1}\right\|_{1},\|T\|_{\Sigma(\bar{A}), \Sigma(\bar{B})} \leq\|T\|$. We shall denote

$$
\|H(T)\|=\|H(T)\|_{H(\bar{A}), H(\bar{B})} .
$$


If $f \in H(\bar{A}),\|f\|$ will be the norm of $f$ in $H(\bar{A})$.

For a given interpolator $\Phi$ we define

$$
\bar{A}_{\Phi}=\Phi_{\bar{A}}(H(\bar{A})), \quad\|a\|_{\Phi}=\inf \left\{\|f\|_{;} a=\Phi_{\bar{A}}(f)\right\}
$$

and, for a fixed $c=c_{\ddot{A}}>1$, we can associate to every $a \in \vec{A}_{\Phi}$ an element $h_{a} \in H(\bar{A})$ such that $\Phi_{\bar{A}}\left(h_{a}\right)=a$ and $\|a\|_{\Phi} \leq\left\|h_{a}\right\| \leq c\|a\|_{\Phi}$. The we say that

$$
a \in \bar{A}_{\Phi} \mapsto h_{a} \in H(\bar{A}) \quad(\bar{A} \in \mathcal{C})
$$

is an almost optimal election.

As for the complex method (1), with the same easy proof, it follows from (8) that $\bar{A} \mapsto \bar{A}_{\Phi}$ is an interpolation method:

Theorem 1 For any interpolator $\Phi$ and $T \in \mathcal{L}(\bar{A} ; \bar{B}), T: \bar{A}_{\Phi} \longrightarrow \bar{B}_{\Phi}$ with $\|T\|_{\Phi} \leq\|H(T)\|$.

A couple of interpolators will be a pair $(\Phi, \Psi)$ of interpolators on the same function spaces $H(\bar{A})$, and

$$
\bar{A}_{\Psi,(\Phi)}=\left\{a=\Psi_{\bar{A}}(f) ; f \in H(\bar{A}), \Phi_{\bar{A}}(f)=0\right\}=\Psi_{\bar{A}}\left(\operatorname{Ker} \Phi_{\bar{A}}\right)
$$

with $\|a\|_{\Psi,(\Phi)}=\inf \left\{\|f\| ; \Phi_{\bar{A}}(f)=0, a=\Psi_{\bar{A}}(f)\right\}$.

We also have (if $\|T\|_{\Psi,(\Phi)}=\|T\|_{\bar{A}_{\Psi,(\Phi)}, \bar{B}_{\Psi,(\Phi)}}$ )

Theorem 2 For any $T \in \mathcal{L}(\bar{A} ; \bar{B}), T: \bar{A}_{\Psi,(\Phi)} \longrightarrow \bar{B}_{\Psi,(\Phi)}$ with $\|T\|_{\Psi,(\Phi)} \leq$ $\|H(T)\|$.

For a fixed the couple $(\Phi, \Psi)$ of interpolators and $a \in \bar{A}_{\Phi} \mapsto h_{a} \in$ $H(\bar{A})$, an almost optimal election, we define the $\Omega$ operator

$$
\Omega_{\bar{A}} a=\Psi_{\bar{A}}\left(h_{a}\right) \in \bar{A}_{\Psi} \quad(a \in \dot{\bar{A}}) .
$$

Rernark If there is no danger of confusion, very often we supress the subscript $\vec{A}$. We denote $[T, \Omega]=T \Omega_{\bar{A}}-\Omega_{\bar{B}} T$. 
Theorem 3 If $T \in \mathcal{L}(\bar{A} ; \bar{B})$, then $[T, \Omega]: \bar{A}_{\Phi} \longrightarrow \bar{B}_{\Psi,(\Phi)}$ and

$$
\|[T, \Omega] a\|_{\Psi,(\Phi)} \leq C\|a\|_{\Phi} .
$$

Proof. Since $\Phi_{\bar{B}}\left(H(T) h_{a}-h_{T a}\right)=T \Phi_{\bar{A}} f_{a}-\Phi_{\bar{B}} h_{T a}=0$ and $[T, \Omega] a=$ $T \Psi h_{a}-\Psi h_{T a}$, it follows that $[T, \Omega] a \in \bar{B}_{\Psi,(\Phi)}$ and

$\|[T, \Omega] a\|_{\Psi,(\Phi)} \leq\left\|H(T) h_{a}-h_{T a}\right\| \leq c\left(\|H(T)\|\|a\|_{\Phi}+\|T a\|_{\Phi}\right) \leq C\|a\|_{\Phi}$.

For a second almost optimal election $a \mapsto \tilde{h}_{a}$ we have another operator $\tilde{\Omega}$, but $\Omega$ and $\tilde{\Omega}$ are equivalent in the sense that $\tilde{\Omega}-\Omega$ is bounded, since, for any $a \in \bar{A}_{\Phi}, \Phi\left(h_{a}-\tilde{h}_{a}\right)=0$ and $(\tilde{\Omega}-\Omega) a=\Psi\left(h_{a}-\tilde{h}_{a}\right) \in \vec{A}_{\Psi,(\Phi)}$ with

$$
\|(\tilde{\Omega}-\Omega) a\|_{\Psi,(\Phi)} \leq\left\|h_{a}-\tilde{h}_{a}\right\| \leq 2 c\|a\|_{\Phi}
$$

Remark that $\bar{A}_{\Psi,(\Phi)} \leftrightarrow \bar{A}_{\Psi}$ with $\|a\|_{\Psi} \leq\|a\|_{\Psi,(\Phi)}$.

The most interesting examples arise when we have the bounded embedding

$$
\Psi_{\bar{A}}\left(\operatorname{Ker} \Phi_{\bar{A}}\right) \hookrightarrow \operatorname{Im} \Phi_{\bar{A}}
$$

in the sense that, for some constant $C=C(\bar{A})>0$, for every $g \in \operatorname{Ker} \Phi_{\bar{A}}$, there exists $f \in H(\bar{A})$ such that $\Psi(g)=\Phi(f)$ and $\|f\| \leq C\|g\|$. In this case we say that $(\Phi, \Psi)$ is almost compatible and, since then $\bar{A}_{\Psi,(\Phi)} \hookrightarrow \bar{A}_{\Psi}$, we have the following commutator theorem:

Corollary If $(\Phi, \Psi)$ is almost compatible, then

$$
[T, \Omega]: \bar{A}_{\Phi} \rightarrow \bar{B}_{\Phi} \text { and } \tilde{\Omega}-\Omega: \bar{A}_{\Phi} \rightarrow \bar{B}_{\Phi},
$$

and they are bounded operators.

In many important cases we have the "cancelation property"

$$
\Psi_{\bar{A}}\left(\operatorname{Ker} \Phi_{\vec{A}}\right)=\operatorname{Im} \Phi_{\bar{A}}
$$

in the sense that $(\Phi, \Psi)$ is almost compatible and, moreover, for some constant $C=C(\bar{A})>0$, for every $f \in H(\bar{A})$ there exists $g \in \operatorname{Ker} \Phi_{\bar{A}}$ 
such that $\Psi(g)=\Phi(f)$ and $\|g\| \leq C\|f\|$. In this case we say that $(\Phi, \Psi)$ is compatible.

As in [CJMR], we associate to $\Omega$ the twisted direct sums (cf. [Ka])

$$
\bar{A}_{\Phi} \oplus \Omega \bar{A}_{\Phi}=\left\{(a, b) \in \Sigma(\bar{A}) \times \Sigma(\bar{A}) ;\|a\|_{\Phi}+\|\Omega a-b\|_{\Phi}<\infty\right\}
$$

and, to every $T \in \mathcal{L}(\bar{A} ; \bar{B})$, the operator $\tilde{T}(a, b)=(T a, T b)$.

Theorem 4 The following properties are equivalent:

(a) $[T, \Omega]: \bar{A}_{\Phi} \longrightarrow \bar{B}_{\Phi}$ and it is bounded.

(b) $\tilde{T}: \bar{A}_{\Phi} \oplus_{\Omega} \bar{A}_{\Phi} \longrightarrow \bar{B}_{\Phi} \oplus_{\Omega} \bar{B}_{\Phi}$ and it is bounded.

Moreover, if $(\Phi, \Psi)$ is compatible,

$$
A_{\Phi} \oplus \Omega \bar{A}_{\Phi}=\operatorname{Im}\left(\Phi_{\bar{A}}, \Phi_{\bar{B}}\right),
$$

where $\left(\Phi_{\tilde{A}}, \Phi_{\bar{B}}\right) f=\left(\Phi_{\bar{A}} f, \Phi_{\bar{B}} f\right)$.

Over Dom $\Omega_{\bar{A}}=\left\{a \in \bar{A}_{\Phi} ; \Omega_{\bar{A}} \in \bar{A}_{\Phi}\right\}$ we define

$$
\|a\|_{D}=\|a\|_{\Phi}+\left\|\Omega_{\bar{A}}\right\|_{\Phi}
$$

Theorem 5 If $(\Phi, \Psi)$ is compatible, then

(a) Dom $\Omega_{\bar{A}}=\Phi_{\bar{A}}\left(\operatorname{Ker} \Psi_{\bar{A}}\right)$, hence it is a linear subspace of $\vec{A}_{\Phi}$, and

$$
\|a\|_{D} \simeq\left\{\|f\| ; a=\Phi_{\bar{A}}(f), \Psi_{\bar{A}}(f)=0\right\} .
$$

(b) $\bar{A} \mapsto \operatorname{Dom} \Omega_{\bar{A}}$ is an interpolation method.

For the proofs we refer to $[\mathrm{CCS} 1]$.

\section{The complex methods}

Let $S$ and $R$ be two analytic functionals on the strip $\mathbf{S}$, such as $\delta_{\theta}$ and $\delta_{\theta}^{\prime}$. They are linear and bounded on the function spaces $\mathcal{F}(\bar{A})(\bar{A} \in \mathcal{C})$ and, by defining $\mathcal{F}(T) f=T \circ f,(S, R)$ is a couple of interpolators on these function spaces $\mathcal{F}$ : 
Interpolation methods such as

$$
[\bar{A}]_{S}=S(\mathcal{F}(\bar{A})), \quad[\bar{A}]_{R,(S)}=R(\operatorname{Ker} S)
$$

with the natural norms were considered in [Li], [Sc] and [CC1].

For a fixed almost optimal almost optimal election $x \in[\bar{A}]_{S} \mapsto h_{x} \in$ $\mathcal{F}(\bar{A})$ ), the corresponding $\Omega$ operator will be

$$
\Omega_{\bar{A}}^{C}(x)=R\left(h_{x}\right)
$$

and the commutator theorem (Theorem 3 ) reads

$$
\left[T, \Omega_{\bar{A}}^{C}\right]:[\bar{A}]_{S} \longrightarrow[\bar{B}]_{R,(S)}
$$

Let us start with the basic example $\left(\delta_{\theta}, \delta_{\theta}^{\prime}\right)$, with $0<\theta<1$. In [CCS1] we prove:

Theorem 6 it The couple of interpolators $\left(\delta_{\theta}, \delta_{\theta}^{\prime}\right)$ on the function spaces $\mathcal{F}(\bar{A})$ is compatible. Hence

$$
\left[T, \Omega^{C}\right]:[\bar{A}]_{\theta} \longrightarrow[\bar{B}]_{\theta} \quad(T \in \mathcal{L}(\bar{A}, \bar{B}))
$$

and it is bounded (cf $[R W]$ ).

The next results about couples of interpolators $\left(\delta_{\theta}^{(m)}, \delta_{\theta}^{(n)}\right)$ on the spaces $\mathcal{F}(\bar{A})$ are proved in [CCMS].

Theorem $7[\bar{A}]_{\delta_{\theta}^{(n)},\left(\delta_{\theta}\right)}=[\bar{A}]_{\delta_{\theta}^{(n-1)}, \text { and }}$

$$
\left[T, \Omega^{C}\right]:[\bar{A}]_{\theta} \longrightarrow[\bar{B}]_{\delta_{\theta}^{(n-1)}}
$$

a bounded operator.

Theorem 8 Over the class of $L^{p}$ spaces, $\left(\delta_{\theta}^{(n)}, \delta_{\theta}^{(n+1)}\right)$ are almost compatible. Since (cf. /CC1])

$$
\left[L^{p_{0}}, L^{p_{1}}\right]_{\delta_{\theta}^{(n)}}=L^{p}(\log L)^{-n p} \quad\left(\frac{1}{p}=\frac{1-\theta}{p_{0}}+\frac{\theta}{p_{1}}\right)
$$

it follows that

$$
\left[T, \Omega^{C}\right]: L^{p}(\log L)^{-n p} \longrightarrow L^{q}(\log L)^{-n q}
$$

if $T \in \mathcal{L}\left(\left(L^{p_{0}}, L^{p_{1}}\right),\left(L^{q_{0}}, L^{q_{1}}\right)\right)\left(1 / q=(1-\theta) / q_{0}+\theta / q_{1}\right)$. 
Theorem 9 Let $1 \leq p, q<\infty$ be fixed. For weighted $L^{p}$ spaces, $\left(\delta_{\theta}^{(n)}, \delta_{\theta}^{(n+1)}\right)$ is almost compatible. Hence, for any

$$
\begin{gathered}
T \in \mathcal{L}\left(\left(L^{p}\left(\omega_{0}\right), L^{p}\left(\omega_{1}\right)\right) ;\left(L^{q}\left(\omega_{0}\right), L^{q}\left(\omega_{1}\right)\right)\right), \\
{\left[T, \Omega^{C}\right]:\left[L^{p}\left(\omega_{0}\right), L^{p}\left(\omega_{1}\right)\right]_{\delta_{\theta}^{(n)}} \longrightarrow\left[L^{q}\left(\omega_{0}\right), L^{q}\left(\omega_{1}\right)\right]_{\delta_{\theta}^{(n)}} .}
\end{gathered}
$$

Moreover (cf. [CC2]), $\left[L^{p}\left(\omega_{0}\right), L^{p}\left(\omega_{1}\right)\right]_{\delta_{\theta}^{(n)}}=L^{p}\left(\omega_{0}^{1-\theta} \omega_{1}^{\theta} \widehat{\omega}^{-n p}\right)$, with $\widehat{\omega}=$ $1+\left|\log \left(\omega_{0} / \omega_{1}\right)\right|$.

Applications are obtained when explicit almost optimal elections allow to compute the $\Omega$ operator, as it happens in the following instances of the complex methods.

Example 1 For weighted $L^{p}$ spaces of vector valued functions,

$$
\left[L_{0}^{p}\left(\omega_{0} ; A\right), L_{1}^{p}\left(\omega_{1} ; A\right)\right]_{\theta}=L^{p}(\omega ; A) \quad\left(\frac{1}{p}=\frac{1-\theta}{\overline{p_{0}}}+\frac{\theta}{p_{1}}, \omega=\omega_{0}^{1-\theta} \omega_{1}^{\theta}\right),
$$

and

$$
h_{f}(z)=\frac{f}{\|f(\cdot)\|_{A}}\left(\frac{\|f(\cdot)\|_{A}}{\|f\|_{p}}\right)^{\left(\frac{1-z}{p_{0}}+\frac{z}{p_{1}}\right) p}\|f\|_{p}\left(\frac{\omega_{1}}{\omega_{0}}\right)^{\frac{\frac{\omega_{-z}}{p_{0} p_{1}}}{2}}
$$

is an almost optimal election.

Then, for $\left(\delta_{\theta}, \delta_{\theta}^{\prime}\right)$, we have

$$
\Omega^{C} f=h_{f}^{\prime}(\theta)=p\left(\frac{1}{p_{1}}-\frac{1}{p_{0}}\right)\left(\log \frac{\|f(\cdot)\|_{A}}{\|f\|_{p}}\right) f-\frac{p}{p_{0} p_{1}}\left(\log \frac{\omega_{0}}{\omega_{1}}\right) f .
$$

In particular,

$$
\left[L^{p_{0}}, L^{p_{1}}\right]_{\theta}=L^{p}, \quad \Omega^{C} f=f \log \frac{|f|}{\|f\|_{p}},
$$

as in $(6)$, and

$$
\left[L^{p_{0}}\left(\omega_{0}\right), L^{p_{1}}\left(\omega_{1}\right)\right]_{\theta}=L^{p}(\omega), \quad \Omega^{C} f=f \log \frac{\omega_{1}}{\omega_{0}} .
$$


In this case $\Omega^{C}$ is linear.

Example 2 If $\left(A_{n}\right)$ is a sequence of Banach spaces and $\omega_{0}, \omega_{1}$ are two weights on $\mathbf{N}$ (positive integers), then we also have

$$
\left[\ell^{p 0}\left(\omega_{0} ;\left(A_{n}\right)\right), \ell^{p_{1}}\left(\omega_{1} ;\left(A_{n}\right)\right)\right]_{\theta}=\ell^{p}\left(\omega ;\left(A_{n}\right)_{n}\right)
$$

with the similar almost optimal election

$$
h_{u}(z)=\frac{u}{|u|}\left(\frac{|u|}{\|u\|_{p}}\right)^{\left(\frac{1-z}{p_{0}}+\frac{z}{p_{1}}\right) p}\|u\|_{p}\left(\frac{\omega_{1}}{\omega_{0}}\right)^{\frac{p(\theta-z)}{p_{0} p_{1}}}
$$

where $|u|=\left(\left\|u_{1}\right\|_{A_{1}},\left\|u_{2}\right\| A_{A_{2}}, \ldots\right)$ and $h_{u}(z)=\left(h_{u}(z)_{1}, h_{u}(z)_{2}, \ldots\right)$.

For the same couple $\left(\delta_{\theta}, \delta_{\theta}^{\prime}\right)$,

$$
\Omega^{C} u=h_{u}^{\prime}(\theta)=\left\{p\left(\frac{1}{p_{1}}-\frac{1}{p_{0}}\right) \log \frac{|u|}{\|u\|_{p}}-\frac{p}{p_{0} p_{1}} \log \frac{\omega_{0}}{\omega_{1}}\right\} u
$$

Examiple 3 As stated in Theorem 9,

$$
\left[L^{p}\left(\omega_{0}\right), L^{p}\left(\omega_{1}\right)\right]_{\delta_{\theta}^{(n)}}^{j}=L^{p}\left(\omega_{0}^{1-\theta} \omega_{1}^{\theta} \widehat{\omega}^{-n p}\right)
$$

with $\widehat{\omega}=1+\left|\log \left(\omega_{0} / \omega_{1}\right)\right|$. For this space we have the almost optimal election (cf. [CCMS])

$$
h_{f}(z)=\frac{\left(\operatorname{sgn} \log \frac{\omega_{0}}{\omega_{1}}\right)^{n}+\varphi(z)}{1+\left|\log \frac{\omega_{0}}{\omega_{1}}\right|^{n}}\left(\frac{\omega_{0}}{\omega_{1}}\right)^{z-\theta} f
$$

with $\varphi \in \mathcal{H}^{\infty}(\mathbf{S}), \varphi^{(n)}(\theta)=1, \varphi^{(j)}(\theta)=0(j \leq n+1, j \neq n)$.

For the couple $\left(\delta_{\theta}^{(n)}, \delta_{\theta}^{(n+1)}\right), \Omega^{C}$ is the linear operator

$$
\Omega^{C} f=h_{f}^{(n+1))}(\theta)=\frac{n+1+\left|\log \frac{\omega_{0}}{\omega_{1}}\right|^{n}}{1+\left|\log \frac{\omega_{0}}{\omega_{1}}\right|} \log \left(\frac{\omega_{0}}{\omega_{1}}\right) f .
$$




\section{The real methods}

We assume $0<\theta<1$ and $1 \leq p<\infty$. For a given compatible couple $\bar{A}$ we denote $\Delta(\bar{A})=A_{0} \cap A_{1}$ and

$$
J(t ; a)=\max \left(\|a\|_{0}, t\left\|_{1}\right\|_{1}\right) \quad(a \in \Delta(\bar{A}), t>0) .
$$
spaces

The $J-$ method corresponds to the interpolator $\Phi^{J}$ on the function $H^{J}(\bar{A})=\left\{u: \mathbf{R}^{+} \longrightarrow \Delta(\bar{A})\right\}$ measurable; $\left.\|u\|=\left\|t^{-\theta} J(t ; u(t))\right\|_{L^{p}\left(\frac{d t}{t}\right)}<\infty\right\}$ defined as

$$
\Phi_{\bar{A}}^{J}(u)=\int_{0}^{\infty} u(t) \frac{d t}{t} \quad(\Sigma(\bar{A})-\text { valued })
$$

Again, $H^{J}(T)=T \circ u$ if $T \in \mathcal{L}(\bar{A} ; \bar{B})$.

In this case,

$$
\bar{A}_{\Phi^{J}}=\bar{A}_{\theta, p}=\left\{a \in \Sigma(\bar{A}) ; a=\int_{0}^{\infty} u(t) \frac{d t}{t}, u \in H^{J}(\bar{A})\right\}
$$

and we shall consider an almost optimal election

$$
u_{a} \in H^{J}(\bar{A}), \int_{0}^{\infty} u_{a}(t) \frac{d t}{t}=a,\left\|u_{a}\right\| \leq c\|a\|_{\theta, p} .
$$

To define the $\Omega$ operator we need to associate to $\Phi^{J}$ a second interpolator $\Psi^{J}$ on the same function spaces $H^{J}(\bar{A})$. To gess a definition for $\Phi^{J}$, as in [CCMS] we relate the J-method with the complex method through the reiteration formula

$$
\left[\vec{A}_{\theta_{0}, p_{0}}, \bar{A}_{\theta_{1}, p_{1}}\right]_{\lambda}=\bar{A}_{\theta, p}
$$

with $\theta=(1-\lambda) \theta_{0}+\lambda \theta_{1}$. One inclusion is obtained by defining, for a given $a \in \bar{A}_{\theta, p}$,

$$
f_{a}(z)=\int_{0}^{\infty} t^{\left(\theta_{1}-\theta_{0}\right)(z-\lambda)} u_{a}(t) \frac{d t}{t}
$$

and then

$$
\Omega^{C}(a)=f_{a}^{\prime}(\lambda)=\left(\theta_{1}-\theta_{0}\right) \int_{0}^{\infty}(\log t) u_{a}(t) \frac{d t}{t}
$$


Thus, we are led to define

$$
\Psi_{\bar{A}}^{J}(u)=\int_{0}^{\infty}(\log t) u(t) \frac{d t}{t}
$$

In [CCS1] we prove:

Theorem $10\left(\Phi^{J}, \Psi^{J}\right)$ is a compatible couple of interpolators on the function spaces $H^{J}(\bar{A})$ and $\Omega^{J}(a)=\int_{0}^{\infty}(\log t) u_{a}(t) \frac{d t}{t}$.

The $\mathbf{K}$-method is the interpolation method defined by the interpolator

$$
\Phi^{K}\left(a_{0}, a_{1}\right)=a_{0}+a_{1}
$$

on the function spaces

$$
H^{K}(\bar{A})=\left\{\left(a_{0}, a_{1}\right) ; a_{0}(t)+a_{1}(t)=\text { constant, }\left\|\left(a_{0}, a_{1}\right)\right\|<\infty\right\}
$$

where we assume $\left(a_{0}, a_{1}\right): \boldsymbol{R}^{+} \rightarrow A_{0} \times A_{1}$ measurable and .

$$
\left\|\left(a_{0}, a_{1}\right)\right\|=\left\|t^{-\theta}\left(\left\|a_{0}(t)\right\|_{0}+t\left\|a_{1}(t)\right\|_{1}\right)\right\|_{L^{p}\left(\frac{d t}{t}\right)} .
$$

Moreover $H^{K}(T)\left(a_{0}, a_{1}\right)=\left(T \dot{\circ} a_{0}, T \circ a_{1}\right)$.

Then $\bar{A}_{\Phi^{K}}=\bar{A}_{\theta, p ; K}$ and it is known that $\bar{A}_{\theta, p ; K}=\bar{A}_{\theta, p}$ with equivalent norms.

Let be

$$
a_{x}=\left(a_{0}, a_{1}\right) \in H^{K}(\bar{A}), a_{0}(t)+a_{1}(t)=x,\|x\|_{\theta, p} \leq c\left\|\left(a_{0}, a_{1}\right)\right\|
$$

an almost optimal selection for $x \in \bar{A}_{\theta, p}$ (for this $\mathrm{K}$-method).

Again we can look for a good second interpolator $\Psi^{K}$ by observing that in the reiteration result (13), if for any

$$
a=f(\lambda) \in\left[\bar{A}_{\theta_{0}, p_{0}}, \bar{A}_{\theta_{1}, p_{1}}\right]_{\lambda}
$$

we çhoose $f \in \mathcal{F}\left(\bar{A}_{\theta_{0, p}}, \bar{A}_{\theta_{1}, p_{1}}\right)$ almost optimal for the complex method and define

$$
g_{t}(z)=t^{(z-\lambda)\left(\theta_{1}-\theta_{0}\right)} f(z),
$$


then $g_{t}(\lambda)=a$ and

$$
a=\int_{-\infty}^{+\infty} g_{t}(i s) P_{0}(\lambda, s) d s+\int_{-\infty}^{+\infty} g_{t}(1+i s) P_{1}(\lambda, s) d s=a_{0}(t)+a_{1}(t)
$$

with $a_{j}(t) \in \bar{A}_{\theta_{j}, p_{j}}(j=0,1)$. Now we can compute the derivative (as in [CCMS]) and we get

$$
\Omega^{C}(a)=f^{\prime}(\lambda)=\int_{0}^{1} a_{0}(t) \frac{d t}{t}-\int_{1}^{\infty} a_{0}(t) \frac{d t}{t} .
$$

This suggests the definition

$$
\Psi_{\tilde{A}}^{K}\left(a_{0}, a_{1}\right)=\int_{0}^{1} a_{0}(t) \frac{d t}{t}-\int_{1}^{\infty} a_{0}(t) \frac{d t}{t}
$$

and then (cf. [CCS1]),

Theorem $11\left(\Phi^{K}, \Psi^{K}\right)$ is a compatible couple of interpolators on the function spaces $H^{K}(\vec{A})$ and $\Omega_{\vec{A}}^{K}(x)=\int_{0}^{1} a_{0}(t) \frac{d t}{t}-\int_{1}^{\infty} a_{0}(t) \frac{d t}{t}$ if $\left(a_{0}, a_{1}\right)=$ $a_{x}$ is the almost optimal election for $x \in \bar{A}_{\theta, p}$.

Moreover $\Omega^{K}=-\Omega^{J}$ (as in [JRW] and [CJMR]).

The commutator theorem of [JRW] is our Corollary of Theorem 3, applied to the couples of interpolators $\left(\Phi^{J}, \Psi^{J}\right)$ and $\left(\Phi^{K}, \Psi^{K}\right)$.

From [JRW] we also get the following examples:

Example 4 For the K-method also

$$
\left(L^{p}\left(\omega_{0}\right), L^{p}\left(\omega_{1}\right)\right)_{\theta, p}=L^{p}(\omega) \quad\left(\omega=\omega_{0}^{1-\theta} \omega_{1}^{\theta}\right)
$$

in this case with the almost optimal election

$$
\left.h_{f}=\left(\chi_{t} f,\left(1-\chi_{1}\right) f\right), \text { with } \chi_{t}=\chi_{\left\{\omega_{0} \leq t p\right.} \omega_{1}\right\}
$$

and

$$
\Omega^{K} f=\frac{1}{p}\left(\log \frac{\omega_{1}}{\omega_{0}}\right) f
$$

Example 5 Let $p_{0}<p_{1}, 1 / p=(1-\theta) / p_{0}+\theta / p_{1}$ and $1 / \alpha=1 / p_{0}-1 / p_{1}$. For

$$
\left(L^{p_{0}}, L^{p_{1}}\right)_{\theta, q}=L^{p, q}
$$


we have the almost optimal election

$$
h_{f}=\left(\xi_{t} f,\left(1-\xi_{1}\right) f\right), \text { with } \xi_{t}=\chi_{\left\{|f|>f^{*}\left(t^{\alpha}\right)\right\}},
$$

and

$$
\Omega^{K} f=-\frac{1}{\alpha} f \log B(f), \text { with } B(f)(x)=|\{|f|>|f(x)|\}| .
$$

\section{Some applications}

Typical applications are obtained for commutators on $L^{p}$ spaces,

$$
[T, b] f=T(b f)-b T(f),
$$

when $T$ is a Calderón-Zygmund operator and $b \in B M O$ on $\mathbf{R}^{n}$, using the fact that $e^{b}$ is an $A_{p}$-weight if $\|b\|_{B M O}$ is small, and then $T$ is bounded on $L^{p}\left(e^{b}\right)$.

Since $T \in \mathcal{L}\left(\left(L^{p}\left(e^{b}\right), L^{p}\left(e^{-b}\right)\right) ;\left(L^{p}\left(e^{b}\right), L^{p}\left(e^{-b}\right)\right)\right)$ and in this case $\Omega^{C} f=b f$ (Example 1), the well known result of [CRW]

$$
[T, b]: L^{p} \longrightarrow L^{p}
$$

follows here from the commutator theorem:

In [CCMS] some weighted variants of (14) are given:

Theorem 12 If $b \in B M O$ and $\alpha \geq 0$, then

$$
[T, b]: L^{p}\left(\frac{1}{1+|b|^{\alpha}}\right) \longrightarrow L^{p}\left(\frac{1}{1+|b|^{\alpha}}\right) .
$$

The proof is an application of the commutator theorem for the couple of interpolators $\left(\delta_{\theta}^{(n)}, \delta_{\theta}^{(n+1)}\right)$.

In the same way, but now for $\left(\delta_{\theta}, \delta_{\theta}^{(n)}\right)$ and for the pair $\left(L^{p_{0}}, L^{p_{1}}\right)$, from the fact

$$
\Omega^{C} \simeq\left(\log \frac{|f|}{\|f\|_{p}}\right)^{n} f
$$

for any $T \in \mathcal{L}\left(\left(L^{p_{0}}, L^{p_{1}}\right) ;\left(L^{p_{0}}, L^{p_{1}}\right)\right)$ in [CCMS] we obtain: 
Theorem 13 If $f \in L^{p}\left(1 / p=(1-\theta) / p_{0}+\theta / p_{1}\right)$, then

$$
T\left(\left(\log \frac{|f|}{\|f\|_{p}}\right)^{n} f\right)-\left(\log \frac{|f|}{\|f\|_{p}}\right)^{n}(T f) \in L^{p}(\log L)^{-(n-1) p} .
$$

In [CCS4] we describe new applications to the commutators of BMO functions with Littlewood-Paley sums and with maximal operators (as in [ST] but only for the unweighted case) by considering the spiaces of vector-valued functions of our examples.

\section{Higher order commutators}

Estimates for higher order commutators were obtained in [Ro] and [CCMS] for the complex method, and in [Mi] for the real method. In [CCS2] we have seen how these higher order commutators theorems extend to our general setting.

To this end, we say that a system of $n$ interpolators $\vec{\Phi}_{n}=\left(\Phi^{1}, \ldots, \Phi^{n}\right)$ on the same function spaces $H(\bar{A})$ is compatible if

$$
\Phi^{k}\left(\operatorname{Ker} \Phi^{1} \cap \cdots \cap \Phi^{k-1}\right)=\operatorname{Im} \Phi^{1} \quad(k=1, \ldots, n)
$$

with bounds (as (10) and (11) in the case $n=2$ ).

Then, for $k=1, \ldots, n$, we set

$$
E_{k}=\left\{\vec{a}=\left(a_{1}, \ldots, a_{k}\right) ; \vec{a}=\vec{\Phi}_{k}(f), f \in H(\bar{A})\right\}
$$

with the natural norm $\|\vec{a}\|_{E_{k}}=\inf \left\{\|f\| ; \vec{\Phi}_{k}(f)=\vec{a}\right\}$.

Moreover, from an almost optimal election $\vec{a} \mapsto h_{\vec{a}}$ for $\vec{a} \in E_{n-1}$,

$$
h_{\vec{a}} \in H(\bar{A}), \bar{\Phi}_{n-1}\left(h_{\vec{a}}\right)=\vec{a},\left\|h_{\vec{a}}\right\| \leq c\|\vec{a}\|_{E_{n-1}},
$$

we define $\Omega_{1}=\Omega$ and

$$
\Omega_{k}(\vec{a})=\Phi^{k+1}\left(h_{\vec{a}}\right) \quad(2<k<n) .
$$

If $T \in \mathcal{L}(\bar{A} ; \bar{B})$, as in [Ro] and [Mi] we define $C_{1}(T)=\left[T, \Omega_{1}\right]$ and

$$
C_{m}(T)=\left[T, \Omega_{m}\right]-\sum_{k=1}^{m-1} \Omega_{k} C_{m-k}(T) \quad(2 \leq m \leq n) .
$$


The main result of [CCS2] is then

Theorem 14 Let $\left(\Phi^{1}, \ldots, \Phi^{n+1}\right)$ be a compatible system of interpolators such that

$$
\left(\Phi^{1}, \ldots, \Phi^{n+1}\right)\left(\operatorname{Ker} \Phi^{1} \cap \cdots \cap \operatorname{Ker} \Phi^{j-1}\right) \subset \operatorname{Im}\left(\Phi^{1}, \ldots, \Phi^{(n+1)-(j-1)}\right)
$$

for every index $j=2, \ldots, n+2$ (with bounds).

Then $C_{n}(T): \bar{A}_{\Phi^{1}} \longrightarrow \bar{B}_{\Phi^{1}}$, bounded, for any $T \in \mathcal{L}(\bar{A} ; \bar{B})$.

The classical complex method and real $J$ and $K$ methods are associated to systems of interpolators $\left(\Phi^{1}, \ldots, \Phi^{n+1}\right)$ which satisfy the hypoteses of Theorem 14. They are:

(a) Complex method:

$$
\Phi^{k} f=\frac{1}{(k-1) !} f^{k-1}(\theta)
$$

(b) J-method:

$$
\Phi^{k}(u)=\frac{1}{(k-1) !} \int_{0}^{\infty}(\log t)^{k-1} u(t) \frac{d t}{t}
$$

(c) K-method:

$$
\Phi^{k}\left(a_{0}, a_{1}\right)=\frac{(-1)^{k-1}}{(k-2) !} \int_{0}^{1}(\log t)^{k-2} a_{0}(t) \frac{d t}{t}-\int_{1}^{\infty}(\log t)^{k-2} a_{1}(t) \frac{d t}{t},
$$

with $\Phi^{1}=\Phi^{K}$ and $\Phi^{2}=-\Psi^{K}$ of Theorem 11 .

From these results, new applications to the boundedness of iterated commutators $[[T, b], b]$ are derived (cf. [CCS2]).

\section{Final remark}

Obviously our construction applies to other interpolation methods, such as the real methods with function parameters. The minimal method of Aronszajn-Gagliardo comes also from an interpolator (cf. [CCS1]). For the maximal method we need the dual construction described in [CCS3]. 


\section{References}

[BL] J. Bergh and J. Löfström, Interpolation spaces. An introduction, Springer Verlag, 1976.

[CC1] M.J. Carro and J. Cerdà, Complex Interpolation and $L^{p}$ Spaces, Studia Math. 99(1991),57-67.

[CC2] M.J. Carro and J. Cerdà, On the Interpolation of Analytïc Families of Operators, In "Interpolation Spaces and Related Topics", Edited by M. Cwikel, M. Milman and R. Rochberg, Israel Math. Proc. 5(1992),21-33.

[CCMS] M.J. Carro, J. Cerdà, M. Milman and J. Soria, Schechter Methods of Interpolation and Commutators, Math. Nachr. 174(1995), 35-53.

[CCS1] M.J. Carro, J. Cerdà and J. Soria, Commutators and interpolation methods, Ark. Mat. 33(1995),199-216.

[CCS2] M.J. Carro, J. Cerdà, and J. Soria, Higher order commutators in interpolation theory, Math. Scand. 77 (1995), 301-319.

[CCS3] M.J. Carro, J. Cerdà, and J. Soria, Commutators and Duality in Interpolation Theory In "Interaction Between Functional Analysis, Harmonic Analysis, and Probability", Edited by N. Kalton, E. Saab, and S Montgomery-Smith, Lecture Notes in Pure and Applied Mathematics, 175(1996), 125-133.

[CCS4] M.J. Carro, J. Cerdà, and J. Soria, Commutators, interpolation and vector function spaces, preprint.

[CJMR] M. Cwikel, B. Jawerth, M. Milman and R. Rochberg, Differential estimates and commutators in interpolation theory, Analysis at Urbana II Proc. Special Year in Modern Analysis, London Math. Soc. Lecture Notes, Cambridge Univ. Press 138(1989) 170-220.

[JRW] B. Jawerth, R Rochberg and G. Weiss, Commutators and other second order estimates in real interpolation theory, Ark. Mat. 24(1986), 191-219. 
[Ka] N.J. Kalton, Nonlinear commutators in interpolation theory, Mem. Amer. Math. Soc. 385, 1988.

[Li] J.L. Lions, Quelques Procédés d'Interpolation d'pérateurs Linéaires et Quelques Applications, Seminaire Schwartz, II (1960-61),2-3.

[Mi] M. Milman, Higher Order Commutators in the Real method of Interpolation, preprint, 1993.

[Ro] R. Rochberg, Higher onder estimates in complex interpolation theory, preprint, 1993.

[RW] R. Rochberg and G. Weiss, Derivatives of analytic families of Banach spaces, Ann. of Math. 118(1983) 315-347.

[Sc] M. Schechter, Complex Interpolation, Comp. Math. 18(1967) 117147.

[ST] C. Segovia and J.L. Torrea, Vector valued commutators and applications, Indiana Univ. Math. J. 38(1989) 959-971.

Departament de Matemàtica Aplicada i Anàlisi

Universitat de Barcelona

E-08071 Barcelona, Spain Recibido: 23 de Mayo de 1996

E-Mail: carro@cerber.mat.ub.es,

cerda@cerber.mat.ub.es, soria@cerber.mat.ub.es 